\title{
Global, Disglobal, Local
}

\author{
VERA PARLAC \\ University of Calgary
}

During the "Cross-Americas: Probing Disglobal Networks" conference, through the paper presentations and panel discussions, we sought insights into the disciplinary, professional and pedagogical territory exposed by the apparent disjunctions between global and local. It was intriguing to note that in almost every conversation the word disglobal was omitted and interchanged for the local. This interchange was spontaneous, reflecting our tendency to slip into the familiar framework of binaries but also our attempt to define the complex space in which the local and global overlap. So, in this short essay, the notions of global and local are examined more closely in order to define the disglobal. Related concepts of homogenization, specificity and the new design opportunities produced through their overlap are considered too.

Global - when used as a frame of reference for ideas (as in "global idea") refers to something that looks at the world and draws from its diversity. On the other hand, global, when used in the context of economy or power and especially within the neoliberal framework, refers to homogenization, unification, and subjugation to the unifying idea or ideology.

Local - when used in reference to a craftsmanship or manufacturing adds authenticity and ethical value. On the other hand, when used in the context of politics could mean narrow-minded and limiting attitude.

We often use global and local in a similar way in very different contexts: global when referring to something that looks out to the world and draws from its diversity and as something that wants to even out and homogenize layers of that same diversity; local when referring to something with authentic values and identity and as something with myopic vision and intolerant views.

What is then disglobal?

Is disglobal about the resistance to global?

Is disglobal about opportunities generated by the global and capacity

to utilize them to produce a difference within the local?

Is disglobal about using the global or local network to produce a

difference?

Is disglobal about a difference?
In their manifesto for an accelerationist politics Williams and Srnicek pointedly delineate limitations of localism to meet challenges of nonlocal "rooted deep in our everyday infrastructure." ${ }^{1}$ If global and local are already always present what is driving their relationship? Is modification of global towards specificity or diffraction of local towards inclusive driven by the difference? Difference as defined by Deleuze has a capacity to drive a process ${ }^{2}$. Delanda ${ }^{3}$ illustrates this by describing the interaction of intensive properties (hot and cold) of the air as they produce the turbulences and morphogenetic variety of the sky. For him the difference (or the exchange driven by the difference) is a power at the root of a dynamic change.

There are pathways of economy and power that strive to make their own work easier, and therefore gravitate towards homogenization. But there are also pathways of creativity and ideas that thrive in diverse and varied contexts and draw their specificities from them. The specificity of the context drives out the possibility of difference. The perceived conflicts not only between global and local but also between frameworks in which they could be used seem to suggest emergence of new pedagogical and professional configurations. Could this be a space in which disglobal is at work?

Often as a correction to the prevalent homogenization we focus on localness hoping that its specificity will counteract the relentless forces of commercialization, branding and sameness caused by globalization. Globalization generated a new sensitivity to local and, as Sarah Williams Goldhagen points out, a commitment to defend "distinctiveness of societies and cultures"4. Interestingly, instead of neutralizing the effects, the emphasis on locale and the resistance to it seems to generate turbulences and raptures that open new spaces and territories for action. How do we as designers consider these disturbances caused by global processes (and technologies) encountering local contexts? How do we identify the spaces for action? What new strategies and approaches we could use to operate within the disrupted contexts?

Many presentations at the conference addressed, directly or indirectly, these complexities. What stood out were the ways in which designers and communities appropriated and in some cases disrupted manifestations of the global forces of homogenizations (technologies, infrastructures and representations) in search of a new ground, new way of working and new expression. 
Digital technology was one of the prevalent subjects in the work presented. We are all familiar with its unifying effect evident in projects coming from the schools of architecture (and the profession) all around the world. Digital tools and techniques, however, have also been instrumental in democratizing design by allowing new local expression while enabling global access. Several presentations explored how digital technologies, techniques, data and capacity for dynamic simulation can open up new design strategies and processes.

Infrastructure, as another global top down system, also dominated session conversations. A number of presentations showed the work that attempts to capitalize on the network properties of infrastructures while, at the same time, rewiring them for the use by the local communities. This resulted in creation of new tools to research these contexts or new representations that reveal often invisible pathways and processes. The weaving of the global logistics into local workflows gave rise to new methodologies that are not preoccupied with objects. Instead, they produce processes and operate as design catalysts engaging physical, cultural or political environments at variety of scales. Therefore, the words tactics, strategies, trajectories, practices or intensities that appear in topic subtitles, carry that 'active' participatory attitude that design seems to be able to project while engaging with the indigenous contexts. This seems to remove architects from an abstract sphere of operation and place them in a context where they have to engage the new territory modified by 'difference'.

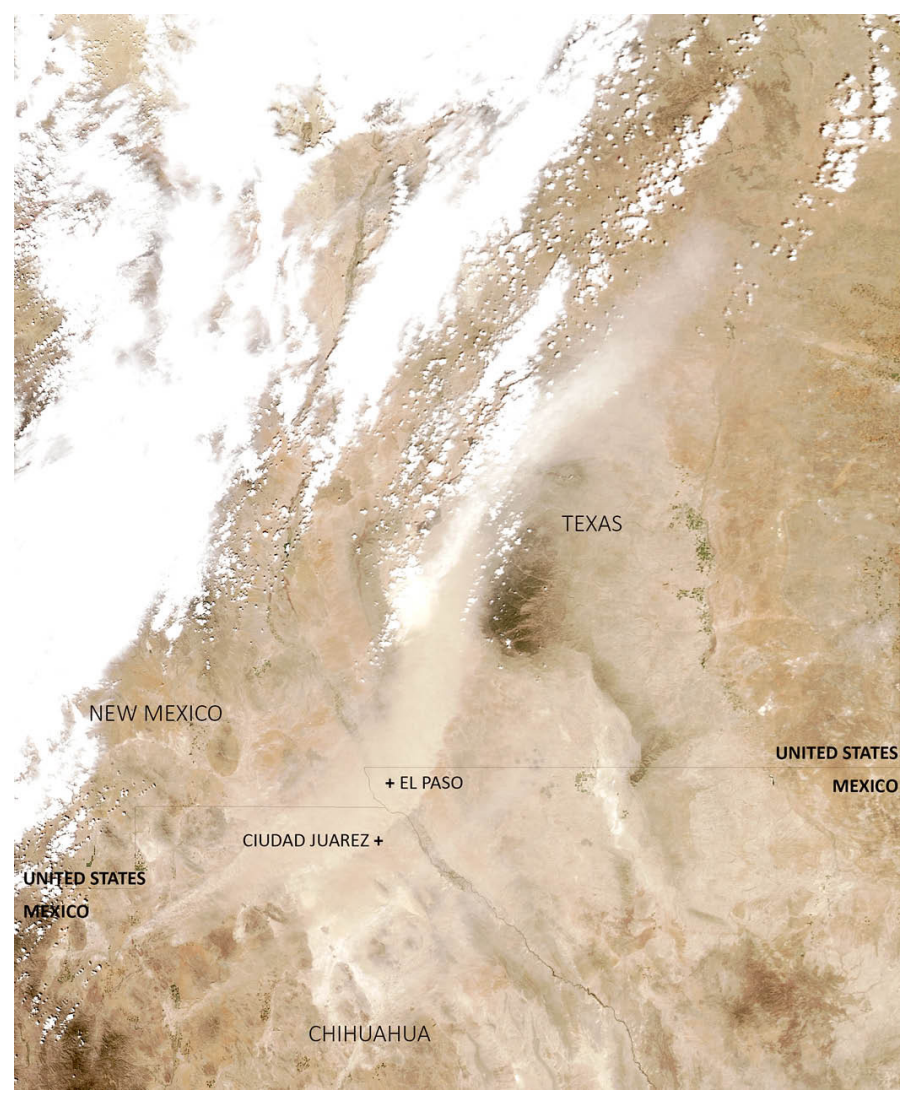

Figure: Project Posters on display at the Pontificia Universidad Católica de Chile, Escuela de Arquitectura courtyard.

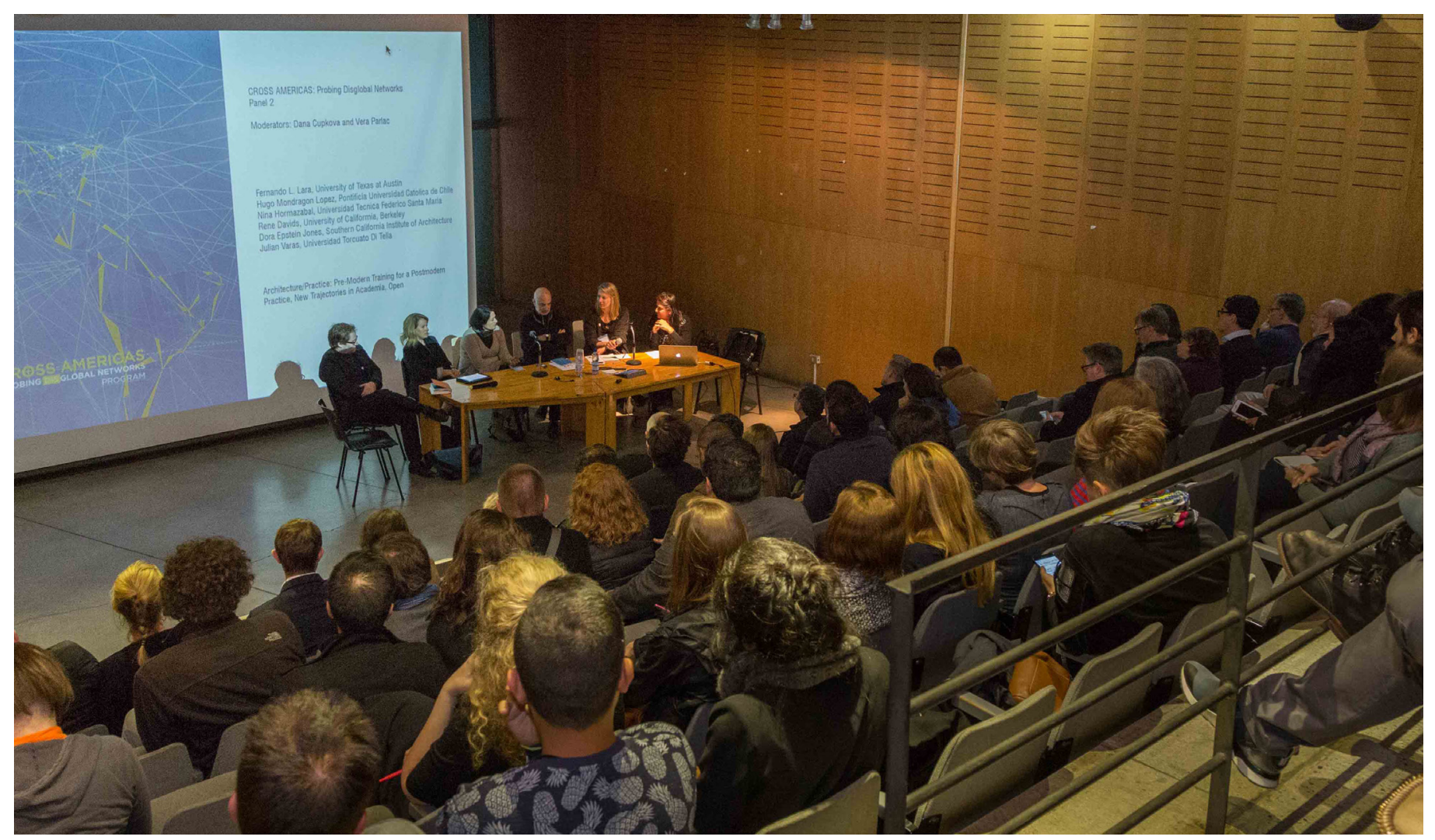

Figure 1: Opening panel session with co-chairs. 
The new information territories and new representations brought forward by the new methodologies highlight disciplinary overlaps; by doing that they expand the sphere of influence available to designers (architects, urban designers and landscape architects). Blurring disciplinary boundaries increases a capacity of the profession to engage complex problems found in the space of exchange between the global and local intensities at the tactical level.

Discussion about disglobal might not be about the mixture of global and local or about identifying within those mixtures an identity or authenticity. It might be about trying to understand how constructed (dynamic) systems work. Ideas about ecology present in the design culture are particularly helpful in thinking about the nature of complex constructed systems. A number of discussions during the conference underlined how ideas about ecology could reframe our thinking about constructed ecologies and the nature of design. Our interest in ecological thinking has highlighted awareness that we are not external to the systems. Discomfort or disbalance that global/local interchange generates is the recognition of a difference that fuels that exchange. There is always a set of parameters that is creating an opportunity to coopt, rewire or redistribute material and other intensities within an existing ecology. This pressure is what qualifies disglobal.

As specificity of the local and uniformity of the global exchange intensities, new territories emerge with varying concentrations of information. Together they appropriate space for their own dynamic interchanges that inevitably give rise to changed conditions that are neither global nor local. The 'difference' encountered by global/local always changes the way global/local occupies, fragments or delaminates new territories. As Châtelet writes: "If I live on a surface, the world no longer faces me; and alterity is no longer lived as a confrontation." ${ }^{5}$ The immersed, lived experience transforms this new territory into something at the same time familiar and alien. In my view this transformation triggered by the 'difference' offers a productive ground for creative and speculative activities capable of mobilizing local specificity to create productive, dynamic and fecund conditions.

An analogy to a musical dissonance could provide a way of thinking about disglobal. In music consonance and dissonance form a structural contrast. These terms define each other reciprocally; the gradient of the presence of each defines the prevalent character of music. The dissonant chord, however, has one of the tones in a dissonance and it is this tone in particular that needs "resolution". The difference in harmony drives the music forward seeking that resolution. The tension produced by the unstable tone configuration demands movement to a stable chord. In music dissonance is a difference and an "active" moment that demands a motion and invites a change ${ }^{6}$. In general dissonance reflects a conflict or an uncomfortable state in the midst of change. Disglobal, being situated at the convergence of the local and global could in a similar fashion carry a level of discomfort. It could operate as that 'active' moment and be a productive difference that can move potencies over a critical threshold to produce new configuration of forces. Formed by the confluence of homogenization (global) and specificity (local), disglobal could give rise to a new sensibility that learns from the settings and histories it engages; or, as in the movement and interaction of intensive properties, the disglobal, as (intensive) difference, can produce the turbulence and be at the root of dynamic change.

\section{ENDNOTES}

1 Alex Williams and Nick Srnicek, "\#ACCELERATE MANIFESTO for an Accelerationist Politics", Accesed August 1, 2016. http://criticallegalthinking.com/2013/05/14/ accelerate-manifesto-for-an-accelerationist-politics/

2 Gilles Deleuze, Difference and Repetition, (New York: Columbia University Press, 1995)

3 Manuel Delanda, “Intensive and Topological Thinking," Accessed July 15, 2016. https://www.youtube.com/watch?v=0wW2I-nBIDg

4 Sarah Williams Goldhagen, "The Art of a Critical Vision" Architectural Record November 2015, 48

5 Gilles Châtelet, "On a Little Phrase of Riemanns," Accessed July 20, 2016 https:// www.scribd.com/document/258365763/F-Chatelet-Reimann-s-Phrase-pdf, [First published in Analytiques (Psychanalyse-Écritures-Politiques), May 3 1979, 67-75]

Roger Kaimen, Music: An Appreciation, (Boston: McGraw-Hill Higher Education, 2008) 